\title{
Cuadros depresivos y utilización de servicios de salud en mujeres de la isla de Chiloé
}

\author{
RUBÉN ALVARADO(1), JACOBO NUMHAUSER(2) , LUISA GALÁN(3)
}

\section{RESUMEN}

El objetivo de este trabajo es contribuir al conocimiento de lo que sucede en nuestro país respecto de la relación entre depresión y uso de servicios sanitarios, a través de describir la autovaloración del estado de salud mental y del uso de servicios sanitarios generales y especializados, en dos muestras de mujeres adultas de la isla de Chiloé.

Se trata de dos estudios transversales, cuyas muestras fueron extraidas de las mismas zonas de Chiloé, en forma casi simultánea. La muestra poblacional es de tipo aleatoria y está constituida por 153 mujeres y la de consultantes de APS es de tipo accidental y está compuesta por 123 mujeres. Se aplicó una encuesta para valorar la existencia de un cuadro depresivo, la autoevaluación del estado de salud mental y la utilización de diversos servicios sanitarios.

Poco menos de la mitad de las mujeres de ambas muestras considera estar "deprimida" o "ansiosa", y en contraste con una escala estandarizada se aprecian muy pocos falsos negativos y bastantes falsos positivos. Una baja proporción de quienes presentaron un cuadro depresivo recibió atención especializada en el año previo a la entrevista. Una elevada proporción de las mujeres con cuadros depresivos ha estado en contacto con servicios sanitarios, y esta cifra es similar a la descrita por las mujeres sin cuadro depresivo (excepto para servicios de urgencia). Aquellas con trastornos depresivos muestran una mayor cantidad de consultas en diferentes dispositivos de atención en salud, que es más de dos veces a la observada en las mujeres que no tienen un cuadro depresivo.

La autoevaluación del estado de salud mental debe ser utilizada con cuidado para la identificación de casos con cuadros depresivos en la práctica clínica y en la investigación operativa. La elevada proporción de mujeres con cuadros depresivos que acuden a servicios de salud determina una oportunidad para el desarrollo de estrategias de detección y tratamiento. Lo anterior permitiría mejorar el bajo porcentaje de mujeres que reciben algún tratamiento por su cuadro depresivo. Finalmente, las mujeres con cuadros depresivos generan una mayor demanda de consultas sobre el sistema sanitario general, lo que conlleva mayores costos.

Palabras clave: Uso de servicios sanitarios, demanda de atención en salud, depresión.

\footnotetext{
(1) Escuela de Salud Pública. Facultad de Medicina. Universidad de Chile.ralvarado@med.uchile.cl

(2) Unidad de Salud Mental Hospital de Ancud.

(3) Magíster (c) en Salud Pública. Universidad de Chile.
} 


\section{ABSTRACT}

\section{DEPRESSIVE SYMPTOMS AND SIGNS AND USE OF HEALTH SERVICES IN WOMEN FROM CHILOÉ ISLAND}

The objective of this paper is that of contributing to the awareness of what happens in our country with respect to the relation between depression and the use of health services, by means of describing the self-appraisal of the state of mental health and the use of general and specialized health services, in two samples of adult women from Chiloé island.

It involves two transversal studies, the samples of which were extracted from the same area of Chiloé almost simultaneously. The populational sample is of a random type and consists of 153 women, and the APS consultant sample is of an accidental type and consists of 123 women. A survey was applied to value the existence of depressive symptoms and signs, a self-appraisal of the state of mental health, and the use of different health services.

Slightly less than half the women of both samples considered being "depressed" or "anxious", and in contrast with a standardized scale, very few false negatives could be appraised, as well as many false positives. A low proportion of those who presented depressive symptoms had received specialized attention during the year previous to the interview. A high proportion of the women with depressive symptoms has been in contact with health services, and this number is similar to that of women without depressive symptoms (except for emergency services). Those with depressive disorders present a larger amount of visits to outpatient or other health services, which is more than double that observed in women who do not have depressive symptoms and signs. Self-appraisal of the state of mental health must be dealt with carefully for the identification of cases with depressive signs and symptoms in clinical practice and operative research. The high proportion of women with depressive symptoms and signs who resort to health services, determine an opportunity for the development of detection and treatment strategies. This fact would allow for the improvement of the low percentage of women receiving some treatment for their depressive symptoms. Finally, women with depressive symptoms generate a greater demand for medical office, outpatient or emergency service visits, a fact which signifies higher costs.

Key Words: Health services use, health care demands, depression.

\section{INTRODUCCIÓN}

Los trastornos depresivos constituyen una de las principales causas específicas en la carga de enfermedad para todo el mundo ${ }^{1}$, y las proyecciones hacia el año 2020 plantean que su tendencia creciente los ubicará en el segundo lugar con un 5,7\% del total de Avad perdidos ${ }^{2}$. Su elevada prevalencia poblacional, su tendencia a la cronicidad y su asociación con otras condiciones mórbidas lo transforman en un importante problema de salud pública, capaz de producir una elevada demanda sobre los sistemas de atención en salud, con los consecuentes costos asociados.

Es conocido que la utilización de servicios como objeto de estudio es un fenómeno complejo y dinámico ${ }^{3}$, donde interactúan diversos factores que determinan la demanda de atención (características de la persona, características de su red social, tipo de problema de salud, etc.) con la oferta de servicios existente. Además, el uso de servicios no siempre muestra una distribución normal y la característica de esta función de distribución puede variar con el tiempo ${ }^{3}$. Aunque para la recolección de datos se prefiere el uso de registros, se ha reportado que existe una alta concordancia entre éstos y el autorreporte que las personas hacen ${ }^{4}$.

Se sabe que una parte importante de las personas que consulta en atención primaria presenta un trastorno depresivo, llegando a ser de $29,5 \%$ en Santiago de Chile 5 . Sin embargo, la mayoría no consulta directamente por su 
problema afectivo ${ }^{6}$, y en pocos casos el cuadro es diagnosticado por el médico y tratado adecuadamente ${ }^{7-9}$.

Diversos estudios demuestran que las personas que padecen un cuadro depresivo, tienden a tener una mayor utilización de servicios sanitarios y sociales. Este ha sido un hallazgo consistente en los grandes estudios poblacionales de carácter transversal realizados en USA y Europa $^{10-13}$. De igual forma, se ha reportado una asociación entre depresión y un gran uso de servicios sanitarios, tanto en estudios transversales ${ }^{14}$, como en estudios de casos y controles ${ }^{15}$, así como en estudios longitudinales ${ }^{16}$.

Se han señalado algunas hipótesis respecto de los posibles mecanismos que pudieran estar involucrados. Una de ellas es que existen características de personalidad que predisponen al desarrollo de un cuadro depresivo a la vez que a una mayor utilización de servicios ${ }^{17}$. Otra afirma que es el distrés emocional el que subyace a la depresión y a la búsqueda de atención en salud, fundándose en la contundente evidencia que asocia distrés y uso de servicios 4 y18.

Finalmente, se ha planteado que las personas que padecen una depresión tienen una mayor predisposición a sentir dolor (dada la existencia de mecanismos neurofisiopatológicos comunes entre depresión y dolor), lo que a su vez motivaría la búsqueda de atención médica ${ }^{19}$.

Esto último es consistente con la elevada frecuencia de cuadros depresivos entre las personas que consultan en medicina general y el hecho de que el trastorno afectivo no sea la causa directa de consulta.

En Chile, la asociación entre depresión y trastornos depresivos ha sido poco estudiada. Las publicaciones existentes se refieren a unas pocas zonas del país, con datos que fueron tomados hace más de una década (habiéndose introducido durante este período importantes cambios en el sistema de atención en salud mental), y donde el análisis se hace sobre la totalidad de los trastornos mentales sin diferenciar los cuadros depresivos ${ }^{20}$. En un estudio transversal de tipo poblacional realizado en la ciudad de Santiago, se señala que no existen diferencias en la proporción de personas que ha utilizado servicios generales de salud, al comparar el grupo que presenta un trastorno mental con el que no lo padece $(46,5 \% \mathrm{v} / \mathrm{s}$ $46,0 \%)$. Sin embargo, una mayor proporción de los primeros ha tenido consultas con profesionales del campo de la salud mental $(16,5 \% \mathrm{v} / \mathrm{s} 8,1 \%)$. En un reporte posterior del mismo grupo de investigación ${ }^{21}$, con una muestra ampliada a cuatro zonas del país, los resultados siguen siendo similares: a) tanto las personas que presentan un trastorno mental como las que no, muestran una igual utilización de servicios sanitarios generales (en contraste con lo reportando en estudios extranjeros); b) las personas que presentan un trastorno mental hacen mayor uso de servicios especializados en estos problemas, y c) poco más de un tercio de las personas que tienen un trastorno mental ha recibido atenciones para este problema.

El objetivo de este trabajo es contribuir al conocimiento de lo que acontece en nuestro país sobre la relación entre depresión y uso de servicios sanitarios, específicamente a través de describir lo que sucede en dos muestras de mujeres adultas de la isla de Chiloé (una de tipo poblacional y la otra de consultantes en atención primaria), sobre la autovaloración del estado de salud mental y del uso de servicios sanitarios generales y especializados.

\section{MATERIAL Y MÉTODO}

Se trata de un estudio transversal que recoge la información sobre uso de servicios de salud en forma retrospectiva referida al año anterior (12 meses previos) y está basado en dos muestras de mujeres: a) la primera de ellas es de tipo poblacional, y b) la segunda está compuesta por mujeres que acuden a la consulta de morbilidad general a postas rurales y consultorios de atención primaria. Todas residen en diferentes zonas de la Isla de Chiloé.

Universo y muestra Este trabajo tiene dos universos de referencia. El primero corresponde a todas las mujeres adultas, de 18 a 64 años, beneficiarias de Fonasa, que viven en la Isla Grande de Chiloé; y el segundo, mantiene las mismas características agregándose el hecho 
que consultan a medicina general en sus correspondientes centros de atención primaria.

La primera muestra fue de tipo aleatoria estratificada y mantiene la proporción urbano/ rural que existe en la isla. Los casos fueron extraídos desde las siguientes zonas: Ancud, Castro, Achao, Quemchi, Dalcahue, Curaco de Vélez, Chonchi, Chacao, Caulin, Linao, Puntra, Manao, Quetalco y Calen. La selección al azar se hizo utilizando la fichas familiares en las zonas rurales y el listado de inscritos para el per cápita en las áreas urbanas. La muestra estuvo constituida por 153 casos, tamaño que permite un nivel de confianza del $95 \%$ y un tamaño máximo de error de 4\% (considerando una prevalencia cercana al $7 \%$ ).

La segunda muestra fue de tipo accidental, tomada por cuotas proporcionales de acuerdo al tamaño poblacional de cada lugar. Los casos provienen de los centros de atención primaria ubicados en las mismas zonas de donde se extrajo la muestra poblacional. Para este caso, el tamaño de muestra se calculó considerando una prevalencia cercana al 10\% $(\mathrm{p}=0.1)$, con un nivel de confianza del 95\% y un tamaño máximo de error de 5\%. Se eliminaron 15 casos por información incompleta en las encuestas o en el registro médico. La muestra final estuvo constituida por 123 mujeres.

Se cotejaron los casos para evitar que una misma persona estuviera en ambas muestras, situación que no se dio en ningún caso.

En ambos casos, los encuestadores fueron enfermeras, asistentes sociales y técnicos paramédicos, especialmente capacitados para realizar esta encuesta. Los datos para la primera muestra se recogieron entre junio y julio del año 2001, y los de la segunda muestra entre agosto y octubre del mismo año.

En la Tabla 1 se presentan las características de ambas muestras.

El promedio y la mediana de edad se encuentran alrededor de los 40 años para ambas muestras. Respecto del estado civil, en ambas muestran predominan las mujeres casadas (alrededor de 2/3 de ellas), seguidas de las solteras y luego las convivientes.

Respecto del lugar de residencia, alrededor de la mitad de las mujeres viven en áreas urbanas
Tabla 1. Características de las mujeres que conforman la muestra poblacional $(\mathrm{n}=153)$ y la de consultantes por morbilidad general en centros de APS, de la isla de Chiloé $(\mathrm{n}=123)$

\begin{tabular}{|c|c|c|}
\hline Variable & $\begin{array}{c}\text { Muestra } \\
\text { poblacional }\end{array}$ & $\begin{array}{c}\text { Muestra de } \\
\text { consultantes }\end{array}$ \\
\hline \multicolumn{3}{|l|}{ Edad } \\
\hline •promedio (d.e.) & $40,9(10,5)$ & $41,4(10,9)$ \\
\hline -mediana & 39,5 & 40 \\
\hline \multicolumn{3}{|l|}{ Estado civil } \\
\hline - soltera & $14,4 \%$ & $17,1 \%$ \\
\hline -casada & $68,6 \%$ & $64,2 \%$ \\
\hline •conviviente & $9,2 \%$ & $12,2 \%$ \\
\hline •separada & $3,3 \%$ & $3,3 \%$ \\
\hline •viuda & $4,6 \%$ & $3,3 \%$ \\
\hline \multicolumn{3}{|l|}{$\begin{array}{l}\text { Lugar de } \\
\text { residencia }\end{array}$} \\
\hline •urbano & $64,5 \%$ & $44,7 \%$ \\
\hline •rural & $35,5 \%$ & $55,3 \%$ \\
\hline \multicolumn{3}{|l|}{$\begin{array}{l}\text { Escolaridad } \\
\text { alcanzada }\end{array}$} \\
\hline $\begin{array}{l}\text {-sin instrucción } \\
\text { formal }\end{array}$ & $2,6 \%$ & $4,1 \%$ \\
\hline •enseñanza básica & $65,4 \%$ & $68,3 \%$ \\
\hline •enseñanza media & $23,5 \%$ & $22,8 \%$ \\
\hline •enseñanza superior & $8,5 \%$ & $4,9 \%$ \\
\hline $\begin{array}{l}\text { Mediana de años } \\
\text { de estudio }\end{array}$ & 8 & 6 \\
\hline \multicolumn{3}{|l|}{ Actividad laboral } \\
\hline •dueña de casa & $64,5 \%$ & $65,9 \%$ \\
\hline $\begin{array}{l}\text {-trabajo ocasional } \\
\text { remunerado }\end{array}$ & $13,2 \%$ & $13,8 \%$ \\
\hline $\begin{array}{l}\text {-trabajo estable } \\
\text { remunerado }\end{array}$ & $21,1 \%$ & $17,9 \%$ \\
\hline - otra situación & $1,3 \%$ & $2,4 \%$ \\
\hline \multicolumn{3}{|l|}{$\begin{array}{l}\text { Participación } \\
\text { social }\end{array}$} \\
\hline $\begin{array}{l}\text { •ninguna u } \\
\text { ocasional }\end{array}$ & $64,1 \%$ & $45,5 \%$ \\
\hline $\begin{array}{l}\text { - siempre o todo } \\
\text { el tiempo }\end{array}$ & $35,9 \%$ & $54,5 \%$ \\
\hline
\end{tabular}

e igual proporción en áreas rurales. Sin embargo, la muestra de consultantes presenta una proporción menor de residentes en zonas urbanas respecto de la muestra poblacional $(44,7 \% \mathrm{v} / \mathrm{s}$ $64,5 \%$ respectivamente). 
En cuanto al nivel de escolaridad alcanzado, ambas muestras tienen similar distribución: cerca de 2/3 han llegado hasta enseñanza básica y poco más de $1 / 5$ lo ha hecho hasta la enseñanza media. Sin embargo, la muestra poblacional tiene una mediana de años de estudio aprobados que es mayor a la observada en la muestra de consultantes ( $8 \mathrm{v} / \mathrm{s} 6$ respectivamente), lo que podría explicarse por la mayor proporción de mujeres que son residentes en zonas urbanas en la primera muestra, donde es mayor el acceso a instituciones educacionales.

Ambas muestras tienen una distribución similar respecto de la actividad laboral, donde cerca de $2 / 3$ de las mujeres concentran su actividad en la casa y no perciben remuneración adicional.

Finalmente, se observan diferencias respecto del porcentaje de mujeres que mantiene una participación social activa: $35,9 \%$ en la muestra poblacional y $54,5 \%$ en la muestra de consultantes.

Instrumentos utilizados Los instrumentos utilizados en este estudio fueron los siguientes:

a. Una encuesta de datos personales, que explora la información sobre edad, estado civil y situación de pareja, personas con las que vive, número de hijos, nivel de escolaridad, situación laboral, lugar donde vive y participación en organizaciones sociales. Las preguntas están pre-codificadas y fueron sometidas a un proceso de validación semántica previa.

b. Una escala para medir trastornos depresivos, ansiosos y somatoformes ${ }^{22}$, que está compuesta por 35 preguntas pre-codificadas que permiten diferenciar los tres tipos de cuadros (Subescala para Trastornos Depresivos, Subescala para Trastornos Ansiosos y Subescala para Trastornos Somatoformes), y puede ser utilizada en forma dimensional o categorial (en este último se diferencian tres categorías en cada caso: sin cuadro clínico, con cuadro subclínico y con trastorno clínico); que fue construida, validada y estandarizada en nuestro país usando como referencia los criterios diagnósticos DSM III-R para dichos trastornos. Para este trabajo se utilizan los datos provenientes de la Subescala de Trastornos Depresivos y en algunos de los análisis se engloba en el concepto de "cuadro depresivo" a los cuadros subclínicos y a los trastornos depresivos en forma conjunta.

c. Una encuesta que solicita la autoevaluación del estado de salud mental y el uso de servicios de salud (públicos y privados) en los 12 meses previos a la entrevista, diferenciando el tipo de servicio al que acude y la cantidad de veces en que lo hizo. Para el análisis de los datos en la muestra de consultantes en APS no se consideró la consulta donde se realizó la entrevista.

Análisis de los datos Para la clasificación de los casos se utilizaron las categorías de la Subescala para Trastornos Depresivos ${ }^{22}$. Para los estudios de asociación se realizó análisis de varianza acompañado de comparaciones múltiples (o prueba de Kruskal-Wallis cuando correspondía) y prueba de chi cuadrado, dependiendo del tipo de variables que se incorporaban al análisis.

Todo el análisis estadístico se realizó en EPI INFO 6.04.

\section{RESULTADOS}

\section{Autoevaluación del estado emocional}

En la tabla 2 se muestra la autoevaluación del estado emocional, que corresponde a la respuesta frente a la pregunta: "¿se considera actualmente 'deprimida' o 'ansiosa'?".

En la muestra poblacional un 20,9\% responde que "no" y un $37,9 \%$ que "sólo un poco", lo que suma un $58,8 \%$ del grupo estudiado. Por tanto, un $41,2 \%$ responde en forma afirmativa: $22,9 \%$ dice que "sî" y 18,3\% que "sí, mucho".

Con la finalidad de ver si la autoevaluación se relacionaba con los resultados en la subescala de depresión se procedió a comparar la respuesta sobre autoevaluación con la puntuación obtenida y la clasificación que se deriva de esta. Respecto de lo primero (Tabla 2), si se comparan las 
puntuaciones en la subescala de depresión se puede apreciar que los promedios de cada grupo tienden a crecer progresivamente, desde los que responden "no" hasta los que responden "sí, mucho" $(p<0,0000)$. En el análisis de comparaciones múltiples se encontró que sólo entre el grupo que responde "sólo un poco" y "sî" no existían diferencias estadísticamente significativas $(\mathrm{p}=0,0982)$. Por otra parte, en la Tabla 2 también se puede observar que hay una estrecha relación entre la autoevaluación y la clasificación clínica $(p<0,0000)$ : un 96,9\% de los que responden "no" se ubican en la categoría correspondiente a sin cuadro clínico, situación que sólo ocurre en el $28,6 \%$ de los que responde "sí, mucho", donde un 50,0\% se ubica en la categoría de cuadro subclínico y un $21,4 \%$ en la de trastorno depresivo.

En el caso de la muestra de consultantes de APS (Tabla 2), un 16,3\% respondió "no" a la pregunta, un 35,0\% respondió "sólo un poco" y $48,7 \%$ respondió que "sî́" y "sí, mucho". Esta última cifra es sólo un poco mayor a la observada en la muestra poblacional, indicando que no habrían grandes diferencias respecto de la autoevaluación del estado emocional entre ambos grupos.
Al igual que en el caso anterior, se encontró que la puntuación en la subescala de depresión crecía progresivamente desde el grupo que respondía "no" hasta el que respondía "sí, mucho" ( $p<0,0000)$ (Tabla 2). El análisis de comparaciones múltiples mostró que no había diferencias estadísticamente significativas entre la puntuación de los grupos "no" y "sólo un poco" ( $\mathrm{p}=0.1043)$, así como entre los grupos que responden "sî" y "sí, mucho" $(\mathrm{p}=0,0551)$. También se puede observar que existe asociación entre la autoevaluación y la ubicación de la persona dentro de una categoría clínica: un $100 \%$ de las que responde "no" se clasifican como sin cuadro clínico y sólo un $27,8 \%$ queda en esta categoría entre las que responden "sí, mucho" (Tabla 2, p<0,0000).

En general, se puede afirmar que un poco menos de la mitad de las mujeres se autoevalúa como siendo afectada en su estado emocional (siendo un poco más elevada la proporción en la muestra de consultantes en APS), y con una estrecha asociación entre la autoevaluación y los resultados en un estándar externo como lo es una escala para valorar la existencia de cuadros depresivos.

Tabla 2. Autoevaluación del estado emocional(*) en dos muestras de mujeres de la isla de Chiloé, una de tipo poblacional $(n=153)$ y otra de consultantes en centros de APS $(n=123)$

\begin{tabular}{|c|c|c|c|c|c|c|c|}
\hline \multirow{3}{*}{$\begin{array}{l}\text { Autoevaluación } \\
\text { Muestra } \\
\text { Poblacional }\end{array}$} & \multicolumn{2}{|c|}{ Distribución } & \multicolumn{3}{|c|}{ Clasificación de acuerdo a la escala } & \multicolumn{2}{|c|}{ Puntaje } \\
\hline & \multicolumn{2}{|r|}{$\%$} & \multirow[t]{2}{*}{ Sin cuadro } & \multirow[t]{2}{*}{$\begin{array}{c}\text { Cuadro } \\
\text { subclínico }\end{array}$} & \multirow[t]{2}{*}{$\begin{array}{l}\text { Trastorno } \\
\text { depresivo }\end{array}$} & \multicolumn{2}{|c|}{ prom (de) } \\
\hline & & & & & & & \\
\hline -No & 32 & 20,9 & 96,9 & - & 3,1 & 3,3 & $(4,7)$ \\
\hline -Sólo un poco & 58 & 37,9 & 84,5 & 15,5 & - & 7,8 & $(3,8)$ \\
\hline -Sí & 35 & 22,9 & 71,4 & 22,9 & 5,7 & 9,3 & $(4,8)$ \\
\hline •Sí, mucho & 281 & 8,3 & 28,6 & 50,0 & 21,4 & 15,5 & $(5,3)$ \\
\hline Total & 153 & 100,0 & & & & & \\
\hline $\begin{array}{l}\text { Muestra de } \\
\text { consultantes }\end{array}$ & & & & & & & \\
\hline •No & 20 & 16,3 & 100,0 & - & - & 5,3 & $(2,3)$ \\
\hline -Sólo un poco & 43 & 35,0 & 88,4 & 9,3 & 2,3 & 7,2 & $(4,5)$ \\
\hline •Sí & 42 & 34,1 & 45,2 & 40,4 & 14,3 & 12,5 & $(5,4)$ \\
\hline •Sí, mucho & 18 & 14,6 & 27,8 & 38,9 & 33,3 & 15,7 & $(6,7)$ \\
\hline Total & 123 & 100,0 & & & & & \\
\hline
\end{tabular}

(*) Nota: corresponde a la pregunta: “¿se considera actualmente ‘deprimida’ o ‘nerviosa’?” 


\section{Contacto con servicios especializados}

En la Tabla 3 se presenta la frecuencia con que las mujeres señalaron haber estado en contacto con servicios especializados para tratamiento psiquiátrico y/o psicológico durante el año previo a la entrevista (se considera positivo si el tratamiento está siendo recibido en el momento de ser encuestada), en el caso de ambas muestras estudiadas.

En general, sólo un bajo porcentaje de las personas que presentan un cuadro subclínico o un trastorno depresivo al momento de la entrevista han estado (o están) en contacto con un tratamiento especializado de tipo psiquiátrico y/o psicológico. En la muestra poblacional, han estado en tratamiento un $25,8 \%$ de las que presentan un cuadro subclínico y un $11,1 \%$ de las que tienen un trastorno depresivo, siendo esta última cifra muy cerca de la del promedio del grupo total $(10,5 \%)$.

En la muestra de consultantes de APS las cifras son un poco más elevadas respecto de su propio promedio (7,2\%): un $14,3 \%$ en las que puntúan como un cuadro subclínico y un $30,8 \%$ de las que presentan un trastorno depresivo.

Es interesante notar que en la muestra de consultantes cerca de un tercio de las mujeres que tiene un trastorno depresivo ha estado en contacto con tratamiento psiquiátrico y/o psicológico en el año previo, situación que baja a sólo un $11,1 \%$ con un trastorno depresivo en la muestra poblacional. Esto tiende a confirmar lo señalado en estudios extranjeros en relación a que las mujeres de muestras poblacionales y las de muestras de consultantes, son diferentes respecto de la propensión a buscar ayuda para sus problemas de salud mental.

\section{Uso de servicios sanitarios}

En la Tablas 4 y 5 se muestra la frecuencia de uso de diferentes servicios sanitarios públicos y privados durante el año previo a la entrevista, en ambas muestras.

En la muestra poblacional, durante el año previo, un $41,2 \%$ ha acudido a su posta rural o consultorio de atención primaria; un 15,0\% lo ha hecho a un servicio de urgencia; un $14,4 \%$ ha recibido atención en un centro ambulatorio de un hospital, y un $14,4 \%$ ha ido a una consulta privada. A modo de síntesis, un 54,2\% ha acudido a cualquier dispositivo sanitario del sistema público y un $62,7 \%$ lo ha hecho en cualquier dispositivo sanitario (público y/o privado).

En la Tabla 4 se puede observar que en la muestra poblacional las mujeres con un cuadro depresivo tienden a usar más servicios sanitarios, tanto respecto de la proporción de ellas que lo han hecho al menos una vez, como en relación a la cantidad de veces que esto ha ocurrido. Sin embargo, estas diferencias no fueron estadísticamente significativas, con excepción de los servicios de urgencia. En este dispositivo, un $44,4 \%$ de las mujeres con un trastorno depresivo ha consultado al menos una vez en el

Tabla 3. Antecedente de haber estado en tratamiento psicológico y/o psiquiátrico en el año previo a la entrevista, en dos muestras de mujeres de la isla de Chiloé, una de tipo poblacional $(\mathrm{n}=153)$ y otra de consultantes en centros de APS $(\mathrm{n}=123)$

\begin{tabular}{|c|c|c|c|c|}
\hline \multirow{3}{*}{$\begin{array}{l}\text { Presencia de un } \\
\text { cuadro depresivo }\end{array}$} & \multicolumn{4}{|c|}{ Antecedente de haber estado en tratamiento } \\
\hline & & & & \\
\hline & $\mathbf{n}$ & $\%$ & $\mathbf{n}$ & $\%$ \\
\hline $\begin{array}{l}\text { Muestra poblacional } \\
\text {-Sin cuadro clínico }\end{array}$ & 106 & 93,8 & 7 & 6,3 \\
\hline -Cuadro subclínico & 23 & 74,2 & 8 & 25,8 \\
\hline - Trastorno depresivo & 8 & 88,9 & 1 & 11,1 \\
\hline Total & 137 & 89,5 & 16 & 10,5 \\
\hline $\begin{array}{l}\text { Muestra de consultantes } \\
\text { - Sin cuadro clínico }\end{array}$ & 81 & 98,8 & 1 & 1,2 \\
\hline •Cuadro subclínico & 24 & 85,7 & 4 & 14,3 \\
\hline - Trastorno depresivo & 9 & 69,2 & 4 & 30,8 \\
\hline Total & 114 & 92,7 & 9 & 7,2 \\
\hline
\end{tabular}


Tabla 4. Uso de servicios sanitarios en el año previo a la entrevista, según tipo de dispositivo y presencia de un cuadro depresivo, en una muestra poblacional $(\mathrm{n}=153)$, de la isla de Chiloé

\begin{tabular}{|c|c|c|c|c|}
\hline \multirow[b]{2}{*}{ Tipo de servicio } & \multicolumn{4}{|c|}{ Presencia de un cuadro depresivo } \\
\hline & $\begin{array}{l}\text { Sin cuadro } \\
\text { clínico }\end{array}$ & $\begin{array}{l}\text { Cuadro } \\
\text { subclínico }\end{array}$ & $\begin{array}{l}\text { Trastorno } \\
\text { depresivo }\end{array}$ & p - value \\
\hline \multicolumn{5}{|l|}{$\begin{array}{l}\text { Porcentaje de personas } \\
\text { que han tenido atención } \\
\text { en ese lugar }\end{array}$} \\
\hline -Posta rural o centro APS & 42,5 & 32,3 & 55,5 & 0,3935 \\
\hline -Servicio de urgencia & 10,6 & 22,6 & 44,4 & 0,0100 \\
\hline -At. ambulatoria hospitales & 13,3 & 19,4 & 11,1 & 0,6658 \\
\hline $\begin{array}{l}\text { - Cualquier servicio } \\
\text { público }\end{array}$ & 51,3 & 61,3 & 66,7 & 0,4568 \\
\hline -Atención privada & 14,2 & 16,1 & 11,1 & 0,9233 \\
\hline $\begin{array}{l}\text { - Cualquier servicio } \\
\text { público y/o privado }\end{array}$ & 61,1 & 67,7 & 66,7 & 0,7682 \\
\hline \multicolumn{5}{|l|}{$\begin{array}{l}\mathbf{N}^{0} \text { de consultas promedio } \\
\text { por persona, en cada lugar } \\
\text { de atención }\end{array}$} \\
\hline - Posta rural o centro APS & $1,1(1,7)$ & $1,7(3,5)$ & $1,6(2,3)$ & 0,5936 \\
\hline -Servicio de urgencia & $0,1(0,5)$ & $0,5(1,5)$ & $2,6(3,7)$ & 0,0032 \\
\hline -At. ambulatoria hospitales & $0,4(1,6)$ & $0,6(2,2)$ & $0,1(0,3)$ & 0,9078 \\
\hline $\begin{array}{l}\text {-Cualquier servicio } \\
\text { público }\end{array}$ & $4,2(4,3)$ & $6,1(8,4)$ & $10,4(9,2)$ & 0,0839 \\
\hline •Atención privada & $0,4(1,8)$ & $0,0(0,2)$ & $0,2(0,6)$ & 0,3819 \\
\hline $\begin{array}{l}\text { - Cualquier servicio } \\
\text { público y/o privado }\end{array}$ & $2,0(2,7)$ & $3,2(4,7)$ & $4,3(5,5)$ & 0,3733 \\
\hline
\end{tabular}

último año (con un promedio de 2,6 consultas), en contraste con el $22,6 \%$ de las que presentan un cuadro subclínico (con un promedio de 0,5 consulta) y un $10,6 \%$ de las que no tienen un cuadro clínico (con un promedio de 0,1 consulta).

En la muestra de consultantes de APS, se encontró que en el año anterior un 62,6\% había acudido a una posta rural y/o un consultorio de APS; un 39,0\% lo había hecho a un servicio de urgencia; un 18,7\% tenía, al menos, una atención en un centro ambulatorio de un hospital, y un 9,8\% había tenido una consulta privada. En síntesis, un 78,9\% ha acudido a algún dispositivo del sistema público y un $79,7 \%$ lo ha hecho en cualquier dispositivo (público y/o privado).

Respecto de la asociación entre uso de servicios sanitarios y la presencia de un cuadro depresivo, se aprecia una situación semejante a la descrita para la muestra poblacional (Tabla
5). También en este caso una mayor proporción de las mujeres que presentan un trastorno depresivo ha hecho uso de servicios sanitarios en el último año, aunque estas diferencias no fueron estadísticamente significativas. De igual forma, las mujeres con un trastorno depresivo tienen una mayor cantidad de consultas que los otros dos grupos, y las que tienen un cuadro subclínico tienen una cifra más elevada que las que no muestran un cuadro clínico. Sin embargo, estas diferencias sólo fueron significativas para las consultas en APS (posta rural y consultorio).

\section{DISCUSIÓN}

Este trabajo es uno de los pocos en nuestro país que ha tenido por objetivo valorar la relación entre la existencia de un problema de salud mental (en este caso cuadros depresivos) y la 
utilización de servicios sanitarios. A su vez, es el único que utiliza una muestra poblacional y una muestra de consultantes en centros de APS, pertenecientes a las mismas áreas geográficas y cuya información fue recogida muy próxima en el tiempo. Esto es de importancia para el objetivo de este trabajo, ya que en epidemiología psiquiátrica es ampliamente reconocido que se trata de dos poblaciones con características diferentes, tal como lo describieran ${ }^{23-24}$ en sus ya clásicas publicaciones al respecto.

Por otra parte, este trabajo tiene algunas limitaciones que requieren ser analizadas antes de proceder a la discusión de los resultados encontrados. La primera limitación se refiere a los tamaños muestrales; dado que son muestras pequeñas comparadas con otros estudios nacionales, los errores $\bullet$ '5f $\bullet^{\bullet}$ '5f en las pruebas de hipótesis tienden a ser más elevados. A pesar de lo cual, los resultados son coincidentes con los descritos para otras zonas del país ${ }^{21}$. La segunda limitación proviene del método basado en preguntar a las personas seleccionadas sobre la utilización de servicios que han hecho durante el año previo a la entrevista, lo que pudiese contener un sesgo de memoria. Este ha sido el mismo método usado en la mayoría de los estudios previos (nacionales y extranjeros), y existe al menos una evidencia que señala que no hay diferencias significativas entre lo descrito por las personas y el registro que hay en sus fichas clínicas ${ }^{4}$, no existiendo antecedentes en el sentido contrario. La tercera limitación corresponde a la necesidad de extender las conclusiones sólo al universo al cual pertenecen las personas que conforman la muestra, a pesar de lo cual es importante constatar que nuestros resultados son consistentes con lo descrito para

Tabla 5. Uso de servicios sanitarios en el año previo a la entrevista(*), según tipo de dispositivo y presencia de un cuadro depresivo, en una muestra de consultantes en centros de APS $(\mathrm{n}=123)$, de la isla de Chiloé

\begin{tabular}{|c|c|c|c|c|}
\hline \multirow[b]{2}{*}{ Tipo de servicio } & \multicolumn{4}{|c|}{ Presencia de un cuadro depresivo } \\
\hline & $\begin{array}{l}\text { Sin cuadro } \\
\text { clínico }\end{array}$ & $\begin{array}{l}\text { Cuadro } \\
\text { subclínico }\end{array}$ & $\begin{array}{l}\text { Trastorno } \\
\text { depresivo }\end{array}$ & p - value \\
\hline \multicolumn{5}{|l|}{$\begin{array}{l}\text { Porcentaje de personas } \\
\text { que han tenido atención } \\
\text { en ese lugar }\end{array}$} \\
\hline - Posta rural o centro APS & 58,5 & 64,3 & 84,6 & 0,1917 \\
\hline -Servicio de urgencia & 36,6 & 39,3 & 58,3 & 0,4951 \\
\hline -At. ambulatoria hospitales & 23,1 & 14,3 & - & 0,1093 \\
\hline $\begin{array}{l}\text { - Cualquier servicio } \\
\text { Público }\end{array}$ & 78,4 & 75,0 & 92,3 & 0,4289 \\
\hline -Atención privada & 12,2 & 3,6 & 7,7 & 0,3998 \\
\hline $\begin{array}{l}\text { - Cualquier servicio } \\
\text { público y/o privado }\end{array}$ & 79,3 & 75,0 & 92,3 & 0,4344 \\
\hline \multicolumn{5}{|l|}{$\begin{array}{l}N^{\circ} \text { de consultas promedio } \\
\text { por persona, en cada lugar } \\
\text { de atención }\end{array}$} \\
\hline -Posta rural o centro APS & $2,5(3,0)$ & $4,1(5,4)$ & $6,3(4,5)$ & 0,0170 \\
\hline -Servicio de urgencia & $1,0(2,1)$ & $0,9(1,4)$ & $4,1(5,8)$ & 0,2084 \\
\hline -At. ambulatoria hospitales & $0,8(2,0)$ & $1,1(3,1)$ & - & 0,1430 \\
\hline $\begin{array}{l}\text {-Cualquier servicio } \\
\text { Público }\end{array}$ & $4,2(4,3)$ & $6,1(8,4)$ & $10,4(9,2)$ & 0,0839 \\
\hline -Atención privada & $0,4(1,8)$ & $0,0(0,2)$ & $0,2(0,6)$ & 0,3819 \\
\hline $\begin{array}{l}\text { - Cualquier servicio } \\
\text { público y/o privado }\end{array}$ & $4,6(4,8)$ & $6,1(8,4)$ & $10,5(9,4)$ & 0,1145 \\
\hline
\end{tabular}

(*) Nota: para el análisis se excluye la consulta en APS que se hizo en el momento de la entrevista. 
otras zonas del país, lo que permite adicionar evidencia en el mismo sentido a la ya existente.

A nuestro parecer, hay cuatro aspectos que son relevantes para la discusión de los resultados encontrados.

El primero corresponde al grado de coincidencia entre la autoevaluación de la mujer con los resultados en la subescala de depresión, ya sea analizada desde una perspectiva dimensional o categorial. En el primer caso, es claro que la puntuación promedio crece progresivamente desde el grupo que señala no estar "deprimida" o "nerviosa" hasta el grupo que refiere estarlo. Y el análisis de comparaciones múltiples permite apreciar que en ambos grupos, hay una diferencia significativa entre los que responden "no" y los que responden "si" (en cualquiera de sus dos categorías). Por otras parte, desde una perspectiva categórica se observa que la autoevaluación tiende a tener un valor predictivo negativo muy elevado y un valor predictivo positivo sólo moderado: cuando una mujer responde que "no" es altamente probable que en la escala sea clasificada como no tendiendo un cuadro depresivo $(96,9 \%$ y $100,0 \%$ respectivamente en cada muestra), y cuando responde que "si" (en sus dos categorías) tiene una probabilidad de 0,52 de tener un cuadro depresivo (subclínico o trastorno) en la muestra poblacional y una probabilidad de 0,60 en la muestra de consultantes de APS. Estos resultados son relevantes para la práctica clínica y para la investigación operativa, donde habría que tener cuidado cuando se utiliza un solo criterio para la identificación y/o definición de caso con cuadro depresivo, ya que existe el riesgo de sobredimensionar la cantidad de casos incluyendo una proporción alta de falsos positivos.

Un segundo aspecto es la constatación de que una baja proporción de las mujeres que presentaban un cuadro depresivo (subclínico o trastorno) tenía el antecedente de haber estado en algún tratamiento psicológico y/o psiquiátrico en el año previo a la entrevista: $22,5 \%$ en la muestra poblacional y $20,5 \%$ en la muestra de consultantes de APS. Si solamente observamos el grupo que obtiene una puntuación correspondiente a un trastorno depresivo los resultados son parecidos, con diferencias entre ambas muestras: $11,1 \%$ en la muestra poblacional y $30,8 \% \%$ en la muestra de consultantes de APS. En síntesis, sólo una proporción menor de las mujeres que requerirían un tratamiento lo están recibiendo, y esto es relevante para la efectividad del sistema sanitario frente a un problema que se ha transformado en una prioridad de salud pública. Nuestros resultados son coincidentes con los descritos por $^{21}$ para la totalidad de los trastornos mentales en otras cuatro zonas del país.

Hay unos pocos casos donde la escala utilizada muestra que no existe un cuadro depresivo y las personas refieren haber estado en contacto con la atención especializada en el año previo. Esto puede tener al menos tres explicaciones: a) que presentó un cuadro depresivo y al momento de aplicar la escala se encontraba en remisión completa; b) que puede haber sido atendida por otro trastorno mental, y c) que puede haber consultado sin haber tenido un cuadro depresivo u otro trastorno mental. Esta última hipótesis puede ser plausible dado el hallazgo de que la autoevaluación muestra un moderado valor predictivo positivo y a su vez puede ser una fuerte motivación para buscar ayuda especializada. La implicancia de esto último corresponde al riesgo de una inadecuada utilización de recursos especializados que lleva a la ineficiencia del sistema de atención sanitaria. Se trata de un aspecto que debe ser explorado en futuras investigaciones.

Un tercer punto que vale la pena resaltar es la elevada proporción de mujeres con un cuadro depresivo que ha tenido contacto con servicios sanitarios: alrededor de $2 / 3$ en la muestra poblacional y $4 / 5$ en la muestra de consultantes. Esto significa que la mayoría de las mujeres con un cuadro depresivo entra en contacto con dispositivos del sistema sanitario, lugar donde podría ser detectadas para luego recibir un tratamiento oportuno y adecuado, todo lo cual determinaría un impacto poblacional positivo sobre este problema. El mayor obstáculo está en la baja capacidad de detección que muestra el sistema sanitario, tal como fue descrito en un reporte previo para la muestra de consultantes de APS $^{2}$. 
El cuarto aspecto corresponde a la discusión acerca de la posible asociación entre la presencia de un trastorno depresivo y una mayor demanda de atención en servicios sanitarios generales.

Existe abundante evidencia extranjera, que utiliza diversos diseños de investigación, que constata esta asociación ${ }^{10-16}$. Sin embargo, los resultados del único estudio reportado en nuestro país, para trastornos mentales en general (siendo los trastornos depresivos los más prevalentes dentro de todos los que fueron estudiados), no corroboran esta asociación, o sea, no hay diferencias en la utilización de servicios sanitarios generales entre la población que padece un trastorno mental y la que no. Si bien encontramos que no existían diferencias estadísticamente significativas respecto de la utilización de servicios sanitarios generales entre los que no tenían un cuadro clínico y los que si presentaban un cuadro depresivo (con la sola excepción de los servicios de urgencia en la muestra poblacional), creemos que es importante profundizar en los datos y en la forma en que se hace el análisis, antes de arribar a las conclusiones.

Si se considera el porcentaje de personas que ha estado en contacto con servicios sanitarios generales, casi no se aprecia diferencias. Este es el método utilizado en el otro estudio nacional ${ }^{21}$. Sin embargo, esta forma de hacer la comparación puede llevarnos a una apreciación errada, ya que una misma persona podría haber tenido una sola consulta o muchas consultas, y se valora de igual manera. Por eso, pensamos que el número de consultas refleja mejor el tamaño de la demanda y se relaciona mejor con los costos que le están asociados.

Desde la perspectiva recién planteada, se puede observar que en ambas muestras las mujeres con un cuadro subclínico tienen un promedio mayor en el número de consultas que las que no padecen un cuadro clínico y a su vez, las que presentan un trastorno depresivo tienen un promedio aún más elevado que los otros dos grupos. Nótese que el promedio de consultas en las mujeres con un trastorno depresivo respecto de las que no tienen un cuadro clínico es 2,15 veces más alto en la muestra poblacional $(4,3 \mathrm{v} / \mathrm{s}$ 2,0 respectivamente) y 2,28 veces más elevado en la muestra de consultantes (10,5 v/s 4,6 respectivamente). Aunque estas diferencias no fueron estadísticamente significativas, vale la pena recordar lo comentado sobre el tamaño de las muestras utilizadas y la menor probabilidad de rechazar la hipótesis nula (error tipo $\bullet ' 5 f$ ).

Por todo lo señalado anteriormente, pensamos que sí existe una mayor demanda en servicios sanitarios generales en personas que presentan un cuadro depresivo respecto de las que no los padecen, y por tanto, determinan un mayor costo para el sistema de salud. En definitiva, creemos que el debate no está cerrado y se debe continuar investigando en estos aspectos.

\section{REFERENCIAS}

1.- WORLD HEALTH ORGANIZATION. Informe sobre la Salud en el Mundo. Salud Mental: nuevos conocimientos, nuevas esperanzas. 2001. WHO.

2.- MURRAY CJ, LÓPEZ AD. Alternative projections of mortality and disability by cause $1990-2020$ : Global Burder of Disease Study. Lancet 1997, 349: 1498 - 1504.

3.- DIEHR P, YAÑEZ D, ASH A, HORNBROOK D, LIN Y. Methods for analyzing health care utilization and cost. Ann Rev Public Health 1999, 20: $125-144$.

4.- TESSLER R, MECHANIC D, DIMOND M. The effect of psychological distress on physician utilization: a prospective study. J Health Soc Behav 1976, 17: $353-364$.

5.- FLORENZANO R, ACUÑA J, FULLERTON C, CASTRO C. Estudio comparativo de frecuencia y características de los trastornos emocionales en pacientes que consultan en el nivel primario de atención en Santiago de Chile. Rev Med Chile 1998, 126: $397-405$.

6.- FLORENZANO R, ACUÑA J, FULLERTON C, LÓPEZ S, AYLWIN W, QUINTEROS M, MARCHANDÓN A. Frecuencia, características y manejo de los pacientes con desórdenes emocionales atendidos en el nivel primario. Rev Chile Neuro Psiquiatría 1995, 33: 60 - 66.

7.- SIMON GE. Can depression be managed appropriately in primary care?. J Clin Psychiatry 1998, 59 (suppl.2): $3-8$.

8.- DAVIDSON JR, MELTZER - BRODY SE. The underrecognition and undertreatment of depression: what is the breadth and depth of the problem?. J Clin Psychiatry 1999, 60 (suppl.7): 4 - 9.

9.- ALVARADO R, NUMHAUSER J, NEVES L. Prevalencia y detección de cuadros depresivos en pacientes adultas de centros de atención primaria, Chiloé. Rev Chil Salud Pública 2004, 8 (3): $126-136$. 
10.-JOHNSON J, WEISSMAN MM, KLERMAN. Service utilization and social morbidity associated with depressive symptoms in the community. JAMA 1992, 267: 1478 - 1483.

11.-KESSLER RC, ZHAO SH, KATZ SJ, KOUZIS AC, FRANK RG, EDLUND M, LEAF PH. Past - year use of outpatient services for psychiatric problems in the National Comorbidity Survey. Am J Psychiatry 1999, 156: 115 - 123.

12. -SPIJKER J, BIJL RV, DE GRAAF R, NOLEN WA. Care utilization and outcome of DSM III-R major depression in the general population. Results from the Netherlands Mental Health Survey and Incidence Study (NEMESIS). Acta Psychiatr Scand 2001, 104: $19-24$.

13.-SCHMITZ N, KRUSE J. The relationship between mental disorders and medical service utilization in a representative community sample. Soc Psychiatry Psychiatr Epidemiol 2002, 37: 380 - 386.

14.-VEDSTED P, FINK P, TOFT SORENSEN H, OLESEN F. Physical, mental and social factors associated with frequent attendance in Danish general practice. A population - based cross - sectional study. Soc Sci Med 2004, 59: 813 - 823.

15.-FORD JD, TRESTMAN RL, STEINBERG K, TENNEN H, ALLEN S. Prospective association of anxiety, depressive and addictive disorders with high utilization of primary, specialty and emergency medical care. Soc Sci Med 2004, 58: 2145 - 2148.

16.-VON KORFF M, ORMEL J, KATON W, LIN E. Diseability and depression among higth utilizers of health care. Arch Gen Psychiatry 1992, 49: 91 - 100.
17.-GOODWIN RD, HOVEN CW, LYONS JS, STEIN MB. Mental health service utilization in the United States. The role of personality factors. Soc Psychiatry Psychiatr Epidemiol 2002, 37: 561 - 566.

18.-GORTMAKER S, ECKENRODE J, GORE S. Stress and the utilization of health services: a time series and cross - sectional analysis. J Health Soc Behav 1982, 23: $25-38$.

19.-GREDEN JF. Physical symptoms of depression: unmet needs. J Clin Psychiatry 2003, 64 (suppl.7): 5 - 11.

20.-VIELMA M, VICENTE B, RIOSECO P, ESCOBAR B, SALDIVIA S, CRUZAT MY COLS. Utilización de servicios de salud en la Provincia de Santiago. Rev Psiquiatría 1994, 9: 203 - 210.

21.-SALDIVIA S, VICENTE B, KOHN R, RIOSECO P, TORRES S. Use of Mental Health Services in Chile. Psychiatric Services 2004, 55: 71 - 76.

22.-ALVARADO R, VERAA, TOLEDO MI, SERRANO F, GATICA CG, NÚÑEZ C, GODOY S, SOTO O, ALFARO J, ASÚN D. Validación de Escalas para medir Trastornos Depresivos, por Ansiedad y Somatoformes. Cuadernos de Psicología $\mathrm{N}^{\circ} 2$, Universidad Diego Portales. Santiago, Chile. 1992.

23.-GOLDBERG D, HUXLEY P. Mental illness in the community. The pathway to psychiatric care. Londres, Tavistock / Routledge, 1980.

24.-GOLDBERG D, HUXLEY P. Common mental disorders. A bio - social model. Londres, Tavistock/ Routledge, 1992.

Usted puede comentar éste y otros artículos publicados en la Revista Chilena de Salud Pública, enviando un correo electrónico a revistasp@med.uchile.cl 